\title{
Quantum Discord for a Central Two-Qubit System Coupled to an $X Y$ Spin Chain with Energy Current
}

\author{
Feng Zhong
}

Received: 26 March 2013 / Accepted: 2 September 2013 / Published online: 2 October 2013

(C) The Author(s) 2013. This article is published with open access at Springerlink.com

\begin{abstract}
We investigate the quantum discord dynamics of a two-qubit system coupled to an $X Y$ spin-chain environment with energy current. We compared dynamical behaviors of quantum discord under different system-environment couplings, the size of the degrees of freedom of the environment, the anisotropy parameter, and the energy current. The results indicate that the energy current can strongly suppress the quantum discord in the weakcoupling region, while it has no obvious effect on the quantum discord in the strong-coupling regime.
\end{abstract}

Keywords Quantum discord · Energy current · Two-qubit system $\cdot X Y$ spin chain

\section{Introduction}

Entanglement is one of the most fascinating features of quantum mechanics and plays a central role in quantum information processing [1-4]. Much progress concerning quantum entanglement in both theory and experiment has been achieved. However, the quantum state of a composite system may contain other types of nonclassical correlations even when it is separable. Quantum discord, introduced by Ollivier and Zurek [5], is the measure of such nonclassical correlations and is defined as the difference between quantum mutual information and classical correlation. Some investigations show that quantum discord is more general than entanglement in describing quantum correlation [6-8]. Owing to its theoretical and practical significance, quantum discord has been studied in a number of contexts [9-12], for example, quantum discord can be responsible for the quantum computational efficiency of deterministic quantum computation with one pure qubit, and the concept of quantum discord has also been generalized to a continuous variable system for Gaussian states [13].

In the real world, a physical system can never be isolated; the interaction between the quantum system and its environment leads to decoherence. This decoherence process is

F. Zhong ( ()

Department of Electronic Engineering, Hunan University of Science and Engineering,

Yongzhou 425100, China

e-mail: fzhonghunan@163.com 
regarded as the main obstacle in the development of quantum technology. Recently, the dynamics of quantum discord under the effect of the Markovian [14] or non-Markovian [15] environment has been explicitly studied. The results indicate that the time evolutions of quantum discord and entanglement behave differently under the effect of the environment. Furthermore, the effect of decoherence induced by a correlated environment on the dynamics of the quantum discord has been investigated $[16,17]$.

Nonequilibrium steady states were studied by considering energy or magnetic currents. The presence of current in a quantum state can be considered as a quantum phase transition (QPT) to a current-carrying phase. Therefore it has been the subject of intense theoretical studies. The QPT in the ground state of the extended $X Y$ model with energy current was investigated by Titvinidze and Japaridze [18]. Furthermore, the effect of energy current on entanglement in the $X Y$ spin chain was studied in Ref. [19]. As a counterpart, the effect of decoherence induced by an $X Y$ spin chain with energy current on quantum discord may be of interest. In this paper, we consider the quantum discord of two qubits coupled to an $X Y$ spin chain with energy current.

\section{Theoretical Model}

We consider a two-qubit system transversely coupled to an environment of general $X Y$ spin chain with energy current. The corresponding Hamiltonian is given by [19]

$$
\begin{aligned}
H & =H_{I}+H_{E}, \\
H_{I} & =\frac{g}{2} J\left(S_{A}^{z}+S_{B}^{z}\right) \sum_{i=1}^{N} S_{i}^{z}, \\
H_{E} & =\sum_{i=1}^{N}\left(J_{x} S_{i}^{x} S_{i+1}^{x}+J_{y} S_{i}^{y} S_{i+1}^{y}\right)-\lambda J^{E},
\end{aligned}
$$

where $H_{I}$ denotes the interaction between the central two qubits and the surrounding spin chain, with $g$ representing their coupling strength. $H_{E}$ describes the self-Hamiltonian of the $X Y$ spin chain with the presence of energy current, with $N$ being the total number of the sites and $J^{E}$ being the global energy current operator [20,21] given by

$$
J^{E}=\sum_{i=1}^{N} \hat{J}_{i}=\sum_{i=1}^{N} J_{x} J_{y}(x z y-y z x)_{i-1, i+1},
$$

where $(\alpha \beta \gamma-\gamma \beta \alpha)_{i-1, i+1}=S_{i-1}^{\alpha} S_{i}^{\beta} S_{i+1}^{\gamma}-S_{i-1}^{\gamma} S_{i}^{\beta} S_{i+1}^{\alpha}$. This "macroscopic" current is added to the environmental spin chain $H^{X Y}$, multiplied by a Lagrange multiplier $-\lambda$. This form of energy current is remarkable, as the local energy current operator $\hat{J}_{i}=$ $h\left(S_{i}^{x} S_{i+1}^{y}-S_{i}^{y} S_{i+1}^{x}\right)$ for the one-dimensional transverse Ising model can be recognized as the Dzyaloshinsky-Moriya interaction in the theory of weak ferromagnetism. The spin operators $S_{A(B)}^{z}$ and $S_{i}^{\alpha}(\alpha=x, y, z)$ are used to describe the central two qubits and the environmental spin chain, respectively, satisfying the commutation relation $\left[S_{i}^{\alpha}, S_{i}^{\beta}\right]=i \delta_{i j} \varepsilon^{\alpha \beta \gamma} S_{i}^{\gamma}$. Because $\left[S_{A}^{z}+S_{B}^{z}, S_{i}^{\alpha}\right]=0$, the operator $g\left(S_{A}^{z}+S_{B}^{z}\right)$, which is a conserved quantity, can be treated as a constant with different values corresponding to its eigenvalues in the twospin subspace: $\xi_{12}= \pm g, \xi_{3}=\xi_{4}=0$, the corresponding eigenstates can be written as 
$\left|\phi_{1}\right\rangle=|00\rangle,\left|\phi_{2}\right\rangle=|11\rangle,\left|\phi_{34}\right\rangle=\frac{1}{\sqrt{2}}(|01\rangle \pm|10\rangle)$. In terms of these two-qubit states, the Hamiltonian (1) can be rewritten as

$$
\begin{aligned}
H & =\sum_{\mu \mu^{\prime}}\left|\phi_{\mu}\right\rangle\left\langle\phi_{\mu}|H| \phi_{\mu^{\prime}}\right\rangle\left\langle\phi_{\mu^{\prime}}\left|=\sum_{\mu \mu^{\prime}}\right| \phi_{\mu}\right\rangle\left\langle\phi_{\mu^{\prime}}\right|\left(\left\langle\phi_{\mu}\left|H_{I}\right| \phi_{\mu^{\prime}}\right\rangle+\left\langle\phi_{\mu}\left|H_{E}\right| \phi_{\mu^{\prime}}\right\rangle\right. \\
& =\sum_{\mu \mu^{\prime}}\left|\phi_{\mu}\right\rangle\left\langle\phi_{\mu^{\prime}}\right|\left(\frac{J}{2} \xi_{\mu^{\prime}}\left\langle\phi_{\mu} \mid \phi_{\mu^{\prime}}\right\rangle+\left\langle\phi_{\mu}\left|H_{E}\right| \phi_{\mu^{\prime}}\right\rangle\right) \\
& =\sum_{\mu}\left|\phi_{\mu}\right\rangle\left\langle\phi_{\mu}\right| \otimes H_{E}^{\left(\xi_{\mu}\right)}
\end{aligned}
$$

where $\left|\phi_{\mu}\right\rangle(\mu=1,2,3,4)$ is the $\mu$ th eigenstate of the operator $g\left(S_{A}^{z}+S_{B}^{z}\right)$ corresponding to the $\mu$ th eigenvalue $\xi_{\mu}$ and $H^{\left(\xi_{\mu}\right)}=H_{E}+1 / 2 J \xi_{\mu} \sum_{i}^{N} S_{i}^{z}$ is the projected environment Hamiltonian. Considering the initial state $|\Psi(0)\rangle=\left|\psi_{A B}(0)\right\rangle \otimes\left|\varphi_{E}(0)\right\rangle$, where $\left|\Phi_{A B}(0)\right\rangle$ is the initial state of the two central spins and $\left|\varphi_{E}(0)\right\rangle$ is the initial state of the environmental spin chain with energy current. The subsequent time evolution of the coupled spin system is determined by the time evolution operator $U(t)=\exp (-i H t)$. After explicitly knowing $|\Psi(t)\rangle=U(t)|\Psi(0)\rangle$, it will be straightforward for us to ascertain the evolution of the reduced density matrix for the two central spins. Thus, the key task is to determine the time evolution operator in a maximally compact form. For this purpose, we use the Jordan-Wigner transformation which maps spins to one-dimensional spinless fermions with creation and annihilation operators $a_{j}^{\dagger}$ and $a_{j}$,

$$
\begin{aligned}
& a_{j}=\exp \left(\pi i \sum_{l=1}^{i-1} S_{l}^{\dagger} S_{l}^{-}\right) S_{j}^{-}, \\
& a_{j}^{\dagger}=S_{j}^{\dagger} \exp \left(-\pi i \sum_{l=1}^{i-1} S_{l}^{\dagger} S_{l}^{-}\right) .
\end{aligned}
$$

Then the dressed environment Hamiltonian $H_{E}^{\left(\xi_{\mu}\right)}$ becomes

$$
\begin{aligned}
H_{E}^{\left(\xi_{\mu}\right)}= & \frac{1}{4}\left\{\sum_{j}^{N}\left[\left(J_{x}-J_{y}\right)\left(a_{j+1} a_{j}+a_{j}^{\dagger} a_{j+1}^{\dagger}\right)+\left(J_{x}+J_{y}\right)\left(a_{j+1}^{\dagger} a_{j}+a_{j}^{\dagger} a_{j+1}\right)\right]\right. \\
& \left.+\sum_{j}^{N} i \lambda J_{x} J_{y}\left(a_{j+1}^{\dagger} a_{j-1}-a_{j-1}^{\dagger} a_{j+1}\right)+J \sum_{j}^{N} \xi_{\mu}\left(1-2 a_{j}^{\dagger} a_{j}\right)\right\} .
\end{aligned}
$$

Now we introduce Fourier transforms of the fermionic operators described by $a_{j}=$ $\frac{1}{\sqrt{N}} \sum_{k} d_{k} e^{i 2 \pi j k / N}$ with $k=-M, \ldots, M$ and $M=(N-1) / 2$ for odd $N$. Using the Bogoliubov transformation, the Hamiltonian $H_{E}^{\left(\xi_{\mu}\right)}$ can be diagonalized in momentum space. The final result is

$$
H_{k}^{\left(\xi_{\mu}\right)}=\sum_{k} \Lambda_{k}^{\left(\xi_{\mu}\right)}\left(\beta_{k, \xi_{\mu}}^{\dagger} \beta_{k, \xi_{\mu}}-\frac{1}{2}\right),
$$

where the energy spectrum $\Lambda_{k}^{\left(\xi_{\mu}\right)}$ is expressed by

$$
\Lambda_{k}^{\left(\xi_{\mu}\right)}=\frac{1}{2}\left[\varepsilon_{k}+\lambda \frac{\gamma^{2}-1}{4} \sin \left(\frac{4 \pi k}{N}\right)\right],
$$


with

$$
\varepsilon_{k}=\sqrt{\left[\cos \left(\frac{2 \pi k}{N}\right)-\xi_{\mu}\right]^{2}+\gamma^{2} \sin ^{2}\left(\frac{2 \pi k}{N}\right)} .
$$

The parameter $\gamma=\left(J_{x}-J_{y}\right) / J$ measures the anisotropy in the in-plane interaction. The corresponding Bogoliubov transformed fermion operators are defined by

$$
\beta_{k, \xi_{u}}=\cos \frac{\theta_{k}^{\left(\xi_{\mu}\right)}}{2} d_{k}-i \sin \frac{\theta_{k}^{\left(\xi_{\mu}\right)}}{2} d_{-k}^{\dagger},
$$

with angles $\theta_{k}^{\left(\xi_{\mu}\right)}$ satisfying

$$
\theta_{k}^{\left(\xi_{\mu}\right)}=\arctan \left[\frac{\gamma \sin \left(\frac{2 \pi k}{N}\right)}{\xi_{\mu}-\cos \left(\frac{2 \pi k}{N}\right)}\right] .
$$

Following these notations, we can derive the time evolution of the quantum states. The time evolution operator with Hamiltonian (8) can be written as

$$
U(t)=\sum_{\mu}^{4}\left|\phi_{\mu}\right\rangle\left\langle\phi_{\mu}\right| \otimes U_{E}^{\left(\xi_{\mu}\right)}(t),
$$

where $U_{E}^{\left(\xi_{\mu}\right)}(t)=\exp \left(-i H_{E}^{\left(\xi_{\mu}\right)} t\right)$ is the projected time evolution operator for the spin chain dressed by the system-environment interaction parameter $\xi_{\mu}$. As a result, the reduced density matrix of the central spins turns out to be

$$
\rho_{A B}(t)=\operatorname{Tr}_{E}|\Psi(t)\rangle\left\langle\Psi(t)\left|=\sum_{\mu, v=1}^{4} c_{\mu} c_{\nu}^{*}\left\langle\varphi_{E}\left|U_{E}^{\dagger\left(\xi_{v}\right)}(t) U_{E}^{\left(\xi_{\mu}\right)}(t)\right| \varphi_{E}\right\rangle\right| \phi_{\mu}\right\rangle\left\langle\phi_{\nu}\right|,
$$

where $c_{\mu}=\left\langle\phi_{\mu} \mid \Phi_{A B}\right\rangle$. The decoherence factor can be readily obtained as

$$
F(t)=\left\langle\varphi_{E}\left|U_{E}^{\dagger\left(\xi_{v}\right)}(t) U_{E}^{\left(\xi_{\mu}\right)}(t)\right| \varphi_{E}\right\rangle .
$$

Obviously, when $\mu=v,\left|F(t)_{\mu v}\right|$ equals to $1 .\left|F(t)_{\mu v}\right|$ can be considered as the amplitude of the overlap of different bases of the system under the considered environment.

Here the initial state of the system is separable from the initial state of the environment. The initial state of the environment $\left|\varphi_{E}(0)\right\rangle$ is assumed to be

$$
|G\rangle_{\xi}=\prod_{k=1}^{M}\left(\cos \left(\theta_{k}^{\xi} / 2\right)|0\rangle_{k}|0\rangle_{-k}+i \sin \left(\theta_{k}^{\xi} / 2\right)|1\rangle_{k}|1\rangle_{-k}\right)
$$

where $|0\rangle_{k}$ and $|1\rangle_{k}$ denote the vacuum and single excitation state of the $k$ th mode $d_{k}$, respectively. Noticing that

$$
\beta_{k, \lambda \mu}=\left(\cos \Theta_{k}^{\left(\xi_{\mu}\right)}\right) \beta_{k, \lambda}-i\left(\sin \Theta_{k}^{\left(\xi_{\mu}\right)}\right) \beta_{-k, \lambda}^{\dagger},
$$

where $\Theta_{k}^{\left(\xi_{\mu}\right)}=\left(\theta_{k}^{\left(\xi_{\mu}\right)}-\theta_{k}^{(\xi)}\right) / 2$ and $\beta_{k, \lambda_{\mu}}, \beta_{k, \lambda}$ are the normal Bogoliubov modes dressed by the system-environment interaction and the pure environment, respectively. The ground state $|G\rangle_{\xi}$ of the Hamiltonian $H_{E}^{\xi}$ can be obtained from the ground state $|G\rangle_{\xi_{\mu}}$ of the qubitdressed Hamiltonian $H_{E}^{\xi_{\mu}}$ by the following relation:

$$
|G\rangle_{\xi}=\prod_{k=1}^{M}\left(\cos \Theta_{k}^{\left(\xi_{\mu}\right)}+i \sin \Theta_{k}^{\left(\xi_{\mu}\right)} \beta_{k, \xi_{\mu}}^{\dagger} \beta_{-k, \xi_{\mu}}^{\dagger}\right)|G\rangle_{\xi_{\mu}} .
$$


From Eq. (15) and the initial vacuum state, we obtain the explicit expression of the decoherence factor as follows:

$$
\begin{aligned}
\left|F_{12}(t)\right|= & \prod_{k>0}\left[1+2 \sin \left(2 \Theta_{k}^{\left(\xi_{1}\right)}\right) \sin \left(2 \Theta_{k}^{\left(\xi_{2}\right)}\right) \sin \left(\Lambda_{k}^{\left(\xi_{1}\right)} t\right) \sin \left(\Lambda_{k}^{\left(\xi_{2}\right)} t\right) \cos \left(\Lambda_{k}^{\left(\xi_{1}\right)} t-\Lambda_{k}^{\left(\xi_{2}\right)} t\right)\right. \\
& -4 \sin ^{2}\left(\Theta_{k}^{\left(\xi_{1}\right)} t-\Theta_{k}^{\left(\xi_{2}\right)} t\right) \sin ^{2}\left(\Lambda_{k}^{\left(\xi_{1}\right)} t\right) \sin ^{2}\left(\Lambda_{k}^{\left(\xi_{2}\right)} t\right) \sin \left(2 \Theta_{k}^{\left(\xi_{1}\right)}\right) \sin \left(2 \Theta_{k}^{\left(\xi_{2}\right)}\right) \\
& \left.-\sin ^{2}\left(2 \Theta_{k}^{\left(\xi_{1}\right)}\right) \sin ^{2}\left(\Lambda_{k}^{\left(\xi_{1}\right)} t\right)-\sin ^{2}\left(2 \Theta_{k}^{\left(\xi_{2}\right)}\right) \sin ^{2}\left(\Lambda_{k}^{\left(\xi_{2}\right)} t\right)\right]^{\frac{1}{2}} .
\end{aligned}
$$

\section{Dynamical Quantum Discord of Two Qubits}

In this study, we assume that the two qubits are initially prepared in the Werner state [22]

$$
\rho_{A B}(0)=\frac{1-p}{4} I_{A B}+p|\psi\rangle\langle\psi|,
$$

where $|\psi\rangle=(|00\rangle+|11\rangle) / \sqrt{2}$ is the maximally entangled state. In the basis spanned by $\{|00\rangle,|11\rangle,|01\rangle,|10\rangle\}$, the reduced density matrix of the two-qubit system at time $t$ takes the form:

$$
\rho_{A B}(t)=\frac{1}{2}\left(\begin{array}{cc}
\frac{1+p}{2} & p F_{12}(t) \\
p F_{12}^{*}(t) & \frac{1+p}{2}
\end{array}\right) \oplus\left(\frac{1-p}{4}\right) I_{2 \times 2} .
$$

For a two-qubit quantum system, the quantum discord qualifying a measure of quantum correlation, which is defined as the difference between the quantum mutual information and the classical correlation [5],

$$
D\left(\rho_{A B}\right)=I\left(\rho_{A B}\right)-C C\left(\rho_{A B}\right) .
$$

Here $I\left(\rho_{A B}\right)$ is the total correlation of two subsystems, given by

$$
I\left(\rho_{A B}\right)=S\left(\rho_{A}\right)+S\left(\rho_{B}\right)-S\left(\rho_{A B}\right),
$$

with $S(\rho)=-\operatorname{Tr}\left(\rho \log _{2} \rho\right)$ being the von Neumann entropy. $C C\left(\rho_{A B}\right)$ is the classical correlation between the two subsystems $A$ and $B$, which is defined as the maximum information one can obtain from $A$ by performing a perfect measurement on $B$. As discussed in Ref. [23], the classical correlation can be expressed as

$$
C C\left(\rho_{A B}\right)=\sup _{\left\{\hat{P}_{B}^{j}\right\}}\left[S\left(\rho_{A}\right)-S\left(\rho_{A B} \mid\left\{\hat{P}_{B}^{j}\right\}\right)\right],
$$

where $\left\{\hat{P}_{B}^{j}\right\}$ is a complete set of orthogonal projectors operated locally on the subsystem $B$ and $S\left(\rho_{A B} \mid\left\{\hat{P}_{B}^{j}\right\}\right)$ is the quantum conditional entropy, with $\left(\rho_{A B} \mid\left\{\hat{P}_{B}^{j}\right\}\right)=\left[\left(\hat{I} \otimes \hat{P}_{B}^{j}\right) \rho_{A B}(\hat{I} \otimes\right.$ $\left.\left.\hat{P}_{B}^{j}\right)\right] / p_{j}$ being the conditional density operator and $p_{j}=\operatorname{Tr}\left[\left(\hat{I} \otimes \hat{P}_{B}^{j}\right) \rho\left(\hat{I} \otimes \hat{P}_{B}^{j}\right)\right]$ denoting the probability with respect to the outcome $j$.

For the state $\rho_{A B}(t)$ of Eq. (22), $S\left(\rho_{A}\right)=S\left(\rho_{B}\right)$, and the four eigenvalues of it are calculated as $\omega_{1,2}=\frac{1+p}{4} \pm \frac{p}{2}\left|F_{12}(t)\right|$ and $\omega_{3,4}=\frac{1-p}{4}$. Then the quantum mutual information of the state $\rho_{A B}(t)$ reads

$$
I\left(\rho_{A B}\right)=-(1+p) \log _{2}\left(\frac{1+p}{4}\right)+\sum_{m=1}^{4} \omega_{m} \log _{2} \omega_{m} .
$$

In order to evaluate the classical correlation $C C\left(\rho_{A B}\right)$, we propose the complete set of orthogonal projectors $\left\{\hat{P}_{B}^{j}=\left|\delta_{j}\right\rangle\left\langle\delta_{j}\right|, j=1,2\right\}$ for a local measurement performed on the subsystem $B$, where 


$$
\begin{aligned}
& \left|\delta_{1}\right\rangle=\cos \zeta|0\rangle+e^{i \zeta} \sin \zeta|1\rangle, \\
& \left|\delta_{2}\right\rangle=e^{-i \zeta} \sin \zeta|0\rangle-\cos \zeta|1\rangle,
\end{aligned}
$$

with $\zeta \in[0, \pi / 2]$ and $\zeta \in[0,2 \pi]$. For both the projective measurements, one has $p_{1}=p_{2}=$ $1 / 2$, so the corresponding reduced density matrices of subsystem $A$ can be calculated as

$$
\begin{aligned}
& \rho_{A}^{1}=\left[\begin{array}{cc}
\frac{1}{2}(1+p \cos 2 \zeta) & \frac{\sin 2 \zeta}{2} p F_{12}(t) e^{i \zeta} \\
\frac{\sin 2 \zeta}{2} p F_{12}^{*}(t) e^{-i \zeta} & \frac{1}{2}(1-p \cos 2 \zeta)
\end{array}\right], \\
& \rho_{A}^{2}=\left[\begin{array}{cc}
\frac{1}{2}(1-p \cos 2 \zeta) & -\frac{\sin 2 \zeta}{2} p F_{12}(t) e^{i \zeta} \\
-\frac{\sin 2 \zeta}{2} p F_{12}^{*}(t) e^{-i \zeta} & \frac{1}{2}(1+p \cos 2 \zeta)
\end{array}\right] .
\end{aligned}
$$

The eigenvalues of $\rho_{A}^{j}$ can be obtained as

$$
\tau_{1,2}^{j}=\frac{1}{2}(1 \pm \eta)
$$

where $\eta=\sqrt{p^{2}\left(\cos ^{2} 2 \zeta+\left|F_{12}(t)\right|^{2} \sin ^{2} 2 \zeta\right)}$ being dependent on the parameter $\zeta$. It is straightforward to obtain the quantum conditional entropy $\sum_{j} p_{j} S\left(\rho_{A}^{j}\right)=f(\eta)$, given by

$$
f(\eta)=-\frac{1-\eta}{2} \log _{2}\left(\frac{1-\eta}{2}\right)-\frac{1+\eta}{2} \log _{2}\left(\frac{1+\eta}{2}\right) .
$$

Thus the classical correlation is given by

$$
C C\left(\rho_{A B}\right)=-\frac{1+p}{2} \log _{2}\left(\frac{1+p}{4}\right)-\min _{\left\{\hat{p}_{j}\right\}}[f(\eta)],
$$

As $f(\eta)$ is monotonically decreasing, we should choose the minimal value of it by taking the maximal value of $\eta$. Obviously, $\eta \leq p \sqrt{\left(\cos ^{2} 2 \zeta+\sin ^{2} 2 \zeta\right)}=p$ for all choices of the parameter $\zeta$, because the decoherence factor $\left|F(t)_{12}\right|$ is always equal to or less than unity. Then, we have the classical correlation

$$
C C\left(\rho_{A B}\right)=-\frac{1+p}{2} \log _{2}\left(\frac{1+p}{4}\right)-f(p) .
$$

Consequently, the quantum discord is given by

$$
D\left(\rho_{A B}\right)=-\frac{1+P}{2} \log _{2}\left(\frac{1+p}{4}\right)+\sum_{m=1}^{4} \omega_{m} \log _{2} \omega_{m}+f(p) .
$$

Firstly we consider the effect of the current driving field $\lambda$ on the time evolution of quantum discord of the two qubits in the weak-coupling region $(g \ll 1)$. In Fig. 1 we plot the quantum discord as a function of the time for different values of $\lambda$ (which takes the values $0.1,5.0,10.0,15.0$ ), with other parameters given by $g=0.1, \gamma=0.4, N=401, p=0.6$. One can see that the quantum discord decays more and more rapid with the increase of the parameter $\lambda$ in a short-time regime, which means that the introduction of the energy current suppresses the quantum discord. For the long-time limit, the quantum discord tends to a stable value $\sim 0.058$.

In order to compare the quantum discord and quantum entanglement, we now discuss the entanglement dynamics of the two qubits. We employ concurrence as the measure of entanglement. For the Werner state of Eq. (21), the concurrence is readily given by [18]

$$
C\left(\rho_{A B}(t)\right)=\max \left[p\left(\left|F_{12}(t)\right|+\frac{1}{2}\right)-\frac{1}{2}, 0\right] .
$$


Fig. 1 The quantum discord as a function of the scaled time $J t$ for different values of the current driving field $\lambda$. Other parameters are set as $g=0.1, \gamma=0.4$, $N=401, p=0.6$

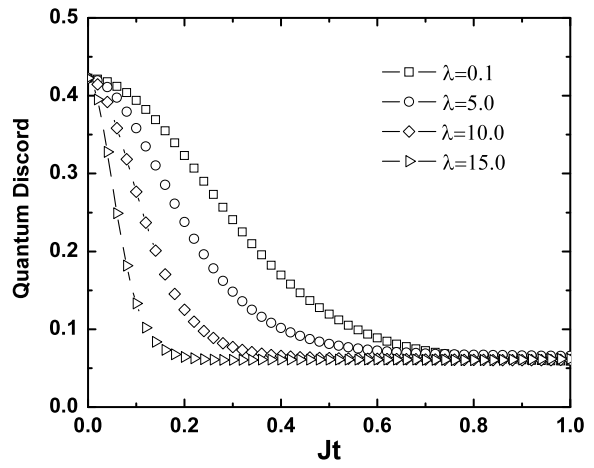

Fig. 2 The concurrence as a function of the scaled time $J t$ for different values of the current driving field $\lambda$. Other parameters are set as $g=0.1, \gamma=0.4$, $N=401, p=0.6$

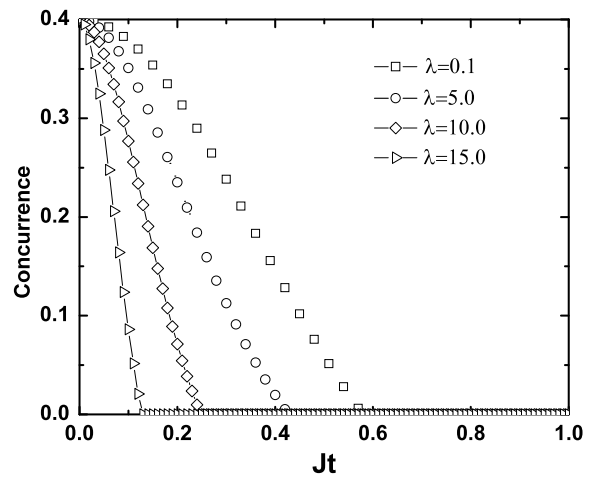

In Fig. 2, we plot the dynamics of concurrence as a function of the scaled time $J t$ under the same condition with that of the quantum discord. One can see that the concurrence vanishes abruptly in finite time, which has been termed entanglement sudden death (ESD). According to Figs. 1 and 2, we can find that ESD occurs for concurrence, whereas the quantum discord is always positive and tends to be a stable value $\sim 0.058$ as the scaled time $J t$ approaches infinity.

Now we check the effects of the anisotropic parameter and the size of degrees of freedom of the environment on the quantum discord in Fig. 3 for the coupling strength $g=0.1$. In Fig. 3(a), the quantum discord is plotted as a function of the scaled time $J t$ for different sizes of spin chain. It shows a phenomenon that increasing the size of the environmental spin chain will decreases the quantum discord. The influence of the anisotropy parameter on the quantum discord is shown in Fig. 3(b). One can find that the quantum discord tends to be a stable value in a finite time and it decreases more rapidly with the increase in the anisotropy parameter.

We now turn to study the effect of the current driving field $\lambda$ on the quantum discord in the strong coupling region $(g \gg 1)$. Figure 4 is a plot of quantum discord as a function of scaled time $J t$ for different values of the current driving field. We adjust the coupling strength from a small value $g=0.2$ to a very large value $g=10.0$. One can see that under weak coupling ( $g=0.2$ ), the quantum discord is suppressed by the energy current to a great degree. As we continue enlarging the coupling strength, the influence of the energy current falls into a decline. When the coupling strength takes a proper value such as $g=10.0$, there will be little difference between the two cases; in other words, the influence of the energy current becomes very feeble in the strong-coupling region. 

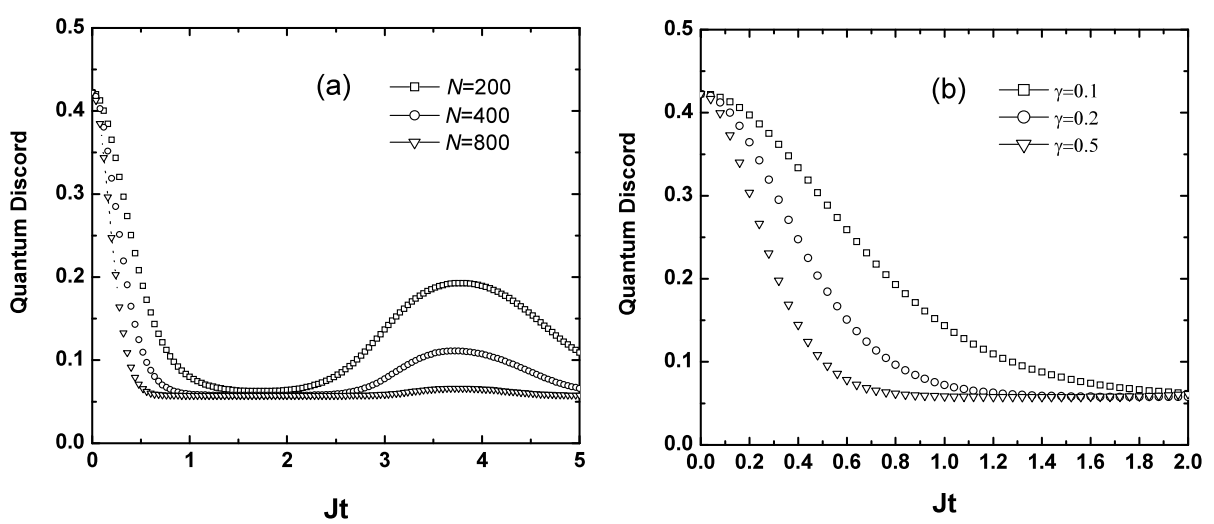

Fig. 3 The quantum discord versus the scaled time $J t$ for (a) $\gamma=0.4$ and (b) $N=401$. Other parameters are set as $g=0.1, \lambda=1, p=0.6$
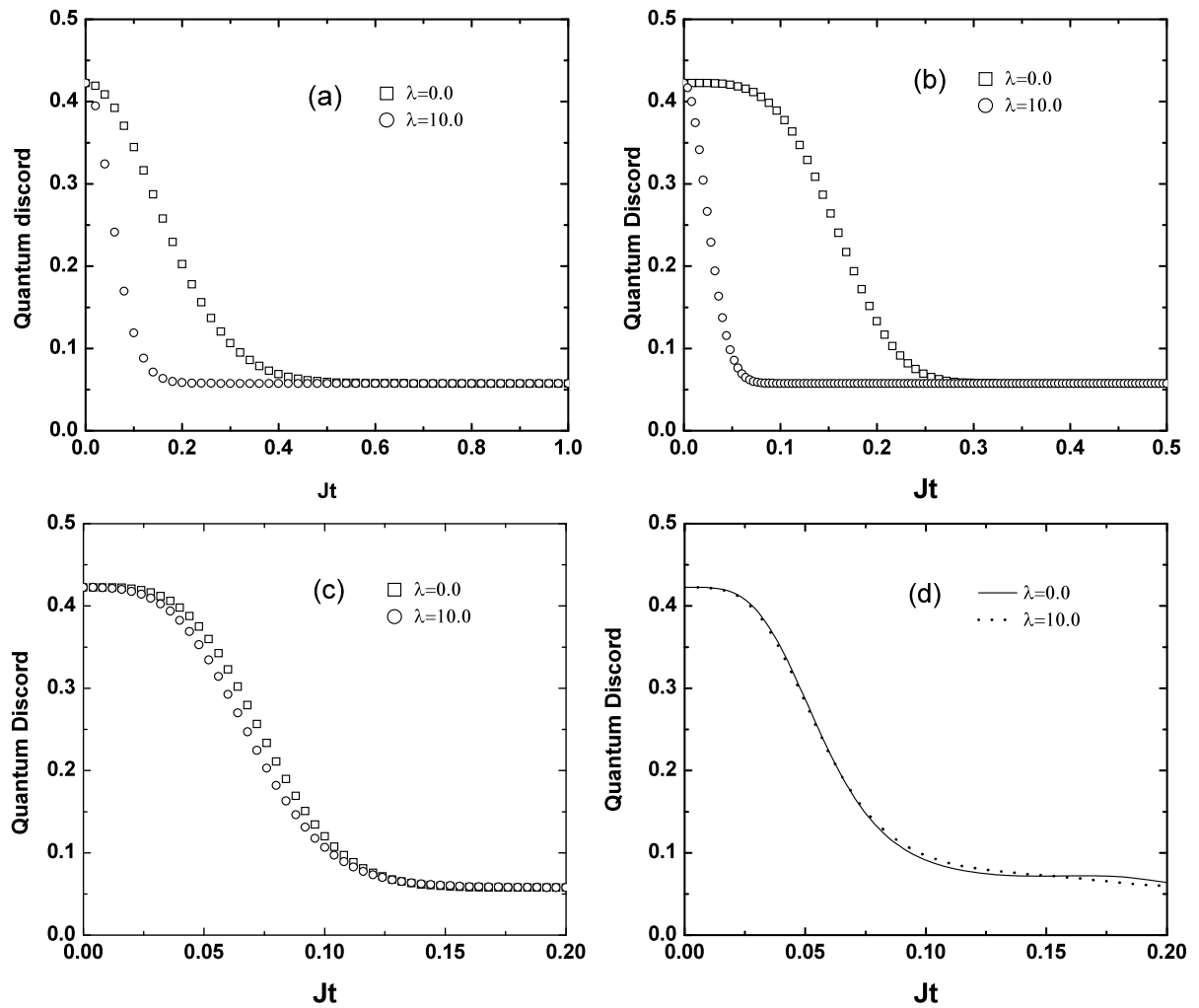

Fig. 4 The quantum discord as a function of the scaled time $J t$ for various coupling strengths: (a) $g=0.2$, (b) $g=1.0$, (c) $g=5.0$, (d) $g=10.0$. The chosen parameters are $\gamma=0.4, N=401, p=0.6$ 


\section{Conclusion}

In summary, we have studied the quantum discord dynamics of a two-qubit system coupled to an $X Y$ spin chain constrained to carry an energy current. By considering the two qubits initially prepared in the Werner state, we have investigated the effects of system-environment coupling strength, the size of degrees of freedom of the environment, the anisotropy parameter, and the energy current on the quantum discord dynamics. It was shown that the energy current can strongly suppress the quantum discord in the weak-coupling regime, whereas it has no obvious effect on the quantum discord in the strong-coupling regime. In the weak-coupling regime, we found that the quantum discord decreases more rapidly with the increase in either the anisotropy parameter or the size of degrees of freedom of the environment. Furthermore, we calculated the entanglement evolution in our model and compare it with the results of the quantum discord under the same condition. We found that entanglement can vanishes abruptly in finite time, while the quantum discord decreases and tends to a finite stable value determined by the initial-state parameter as the scaled time continues.

Open Access This article is distributed under the terms of the Creative Commons Attribution License which permits any use, distribution, and reproduction in any medium, provided the original author(s) and the source are credited.

\section{References}

1. Ficek, Z., Tana's, R.: Phys. Rev. A 74, 024304 (2006)

2. Wang, X.W., Xia, L.X., Wang, Z.Y., Zhang, D.Y.: Opt. Commun. 283, 1196 (2010)

3. Wang, X.W., Zhang, D.Y., Tang, S.Q., Xie, L.J.: J. Phys. B 44, 035505 (2011)

4. Ma, X.S., Wang, A.M., Cao, Y.: Phys. Rev. A 76, 155327 (2007)

5. Ollivier, H., Zurek, W.H.: Phys. Rev. Lett. 88, 017901 (2001)

6. Datta, A., Flammia, S.T., Caves, C.M.: Phys. Rev. A 72, 042316 (2005)

7. Datta, A., Shaji, A., Caves, C.M.: Phys. Rev. Lett. 100, 050502 (2008)

8. Lanyon, B.P., Barbieri, M., Almeida, M.P., White, A.G.: Phys. Rev. Lett. 101, 200501 (2008)

9. Luo, S.: Phys. Rev. A 77, 042303 (2008)

10. Werlang, T., Trippe, C., Ribeiro, G.A.P., Rigolin, G.: Phys. Rev. Lett. 105, 095702 (2010)

11. Chen, Y.X., Yin, Z.: Commun. Theor. Phys. 54, 60 (2010)

12. Hu, M.L., Fan, H.: Ann. Phys. 327, 851 (2012)

13. Giorda, P., Paris, M.G.A.: Phys. Rev. Lett. 105, 020503 (2010)

14. Werlang, T., Souza, S., Fanchini, F.F., Villas Boas, C.J.: Phys. Rev. A 80, 024103 (2009)

15. Wang, B., Xu, Z.Y., Chen, Z.Q., Feng, M.: Phys. Rev. A 81, 014101 (2010)

16. Liu, B.Q., Shao, B., Zou, J.: Phys. Rev. A 82, 062119 (2010)

17. Qiu, L., Wang, A.M.: Phys. Scr. 84, 045021 (2011)

18. Titvinidze, I., Japaridze, G.I.: Eur. Phys. J. B 32, 383 (2003)

19. Liu, B.Q., Shao, B., Zou, J.: Phys. Rev. A 80, 062322 (2009)

20. Tsvelik, A.M.: Phys. Rev. B 42, 779 (1990)

21. Zotos, X., Naef, F., Prelovsek, P.: Phys. Rev. B 55, 11029 (1997)

22. Werner, R.F.: Phys. Rev. A 40, 4277 (1989)

23. Vedral, V.: Phys. Rev. Lett. 90, 050401 (2003) 